\title{
Antibiotic Treatment for Chronic Rhinosinusitis after Endoscopic Surgery: How Long Should Macrolide Antibiotics Be Given?*
}

\author{
Motohiro Sawatsubashi\#, Daisuke Murakami, Shizuo Komune \\ Department of Otolaryngology-Head and Neck Surgery, Graduate School of Medical Sciences, \\ Kyushu University, Fukuoka, Japan \\ Email: "motohiro@qent.med.kyushu-u.ac.jp
}

Received 30 December 2014; accepted 19 January 2015; published 22 January 2015

Copyright (C) 2015 by authors and Scientific Research Publishing Inc.

This work is licensed under the Creative Commons Attribution International License (CC BY).

http://creativecommons.org/licenses/by/4.0/

(c) (i)

Open Access

\section{Abstract}

Background: The purpose of this study was to determine an appropriate period for macrolide antibiotic therapy, and to investigate whether this period could be shorter, for patients with chronicrhino sinusitis (CRS) after functional endoscopic sinus surgery (FESS). Methods: A retrospective analysis of 41 patients undergoing FESS for CRS was performed. All patients underwent pre-operative computed tomography (CT). Patients with fungal sinusitis, allergic fungal sinusitis, and eosinophilic sinusitis were excluded. After FESS, normalized sinus mucosa was confirmed by CT and endoscopy in all patients. Postoperative antibiotic therapy consisted of first-line and second-line regimens. Garenoxacin (GRNX), or clarithromycin (CAM, $400 \mathrm{mg} / \mathrm{day}$ ) was used as the first-line regimens and low-dose macrolide therapy (CAM, $200 \mathrm{mg} / \mathrm{day}$ ) was used as the second-line regimen and was prescribed at outpatient visits based on our clinical criteria. Results: Second-line antibiotic therapy (low-dose CAM) was not necessary in 12 of $41(29 \%)$ patients, while it was prescribed in 29 of $41(71 \%)$. The mean duration of low-dose CAM therapy after FESS was 36 days (range 7 to 122 days; median, 25 days). Patients who received second-line therapy $(n=29)$ were divided into two groups based on the choice of first-line therapy, a GRNX group $(n=13)$ and a non-GRNX group ( $n=16)$. Those in the non-GRNX had longer periods of postoperative CAM therapy than those in the GRNX group. Conclusion: GRNX was associated with a shorter duration of low-dose macrolide therapy after FESS, and $29 \%$ of patients did not need any low-dose macrolide therapy postoperatively. Therefore, macrolide antibiotics should not be routinely prescribed after FESS.

\footnotetext{
${ }^{*}$ This study was partially presented at the $115^{\text {th }}$ Annual Meeting of the ORL Society of Japan, Fukuoka, Japan, 17 May 2014.

"Corresponding author.
} 


\section{Keywords}

\section{Chronic Rhinosinusitis, Functional Endoscopic Sinus Surgery, Macrolide Therapy, Garenoxacin, Postoperative Antibiotic Therapy}

\section{Introduction}

Antibiotics are routinely used by otolaryngologists to reduce the risk of postoperative infection in patients undergoing functional endoscopic sinus surgery (FESS). Antibiotic containing nasal packing can reduce postoperative infections [1]. The efficacy of postoperatively administered antibiotics, including macrolides, for patients with chronic rhinosinusitis (CRS) has been documented [2]. Previous studies have concluded that the sufficient period for low-dose macrolide treatment in CRS patients undergoing FESS should be long-term, i.e., 3 to 6 months [3] [4]. However, consensus on how long patients should be given antibiotics after FESS is not established. In our experience, long-term macrolide administration is not necessary for all patients undergoing FESS for CRS. Furthermore, as antibiotic resistance is now a serious problem; some action is required [5] [6].

The purpose of this study is to examine the state of postoperative antibiotic treatment, especially low-dose macrolide therapy, and to determine the appropriate period of macrolide therapy after FESS for CRS.

\section{Patients and Methods}

The retrospective analysis included 41 patients (28 men, and 13 women) who underwent FESS for CRS at Kyushu University Hospital from April 2009 to October 2013. The mean age at the time of FESS was 59 years, with a range of 14 to 84 years. The diagnosis of CRS was based on a history of rhinosinusitis and the findings of endoscopy and computed tomography (CT) examinations. The patients were screened for allergy and cases with allergy were excluded. There were 20 patients who had sinusitis with polyp and 21 who had sinusitis without polyp. Cases of fungal sinusitis, allergic fungal sinusitis, eosinophil-related fungal rhinosinusitis including allergic fungal rhinosinusitis (AFRS) and eosinophilic sinusitis were not included in this study. All patients included in the study had responded poorly to medical treatments for more than 6 months. Preoperative CT was performed in all cases. The CT findings were summarized according to the Lund-Mackay score [7], which ranges from 0 to 24 (complete opacity of all sinuses), and the severity of sinus mucosal inflammation or fluid accumulation was scored as 0 (complete lucency), 1 (partial lucency), or 2 (completeopacity). The surgeries were performed under general or local anesthesia. All patients underwent FESS without extra-nasal approaches (Caldwell-Luc' procedure or canine fossa approach). The surgical interventions of the procedure were designed to remove the osteomeatal blockage and restore normal sinus ventilation and mucociliary function. The nasal cavity was decongested using gauze with lidocaine and epinephrine; subsequently 0.5 or 1 percent lidocaine with 1:100,000 epinephrine was injected at the level of the middle turbinate root and uncinate process. The uncinated process was removed in cases of middle meatal antrostomy. After widening of the antrostomy for the maxillary sinus, ethmoid cells, frontal sinus, and sphenoid sinus were opened if necessary based on the previous report [8].

Nasal and sinus saline irrigation was performed at the end of the surgery. If patients required nasal packing, the nasal cavity was minimally packed with chitin-coated gauze (Beschitin ${ }^{\circledR}$ F, Unitika Co., Ltd., Kyoto, Japan), that was to be removed within 1 to 2 days after surgery. Septoplasty prior to FESS was performed in 8 patients with severe nasal septal deviation. Nasal and sinus saline irrigation using a bulb syringe was recommended after discharge. Antibiotic prophylaxis (piperacillin 2 g IV or cefazolin 2 g IV) was given during surgery to all patients. Postoperative antibiotic therapy consisted of a first-line regimen and a second-line regimen. Garenoxacin (GRNX, $400 \mathrm{mg} /$ day), or clarithromycin (CAM, $400 \mathrm{mg} /$ day) was used as the first-line regimen during hospitalization, and low-dose CAM (200 mg/day) therapy was used as the second-line regimen during the outpatient period. The surgeon decided whether or not to perform the second-line regimen (low-dose CAM therapy) based on the following conditions;

1) Long-term sinusitis (more than one year);

2) Sinusitis with granulomatous inflammation;

3) Sinusitis with bleeding disorders; 
4) Patients on antiplatelet or anticoagulant treatment;

5) Excessive bleeding during or after FESS;

6) Postoperative nasal packing in place for more than four days;

7) Patients with diabetes mellitus;

8) Patients on steroid treatment for chronic disease.

All patients underwent postoperative CT examination at three or four months after the FESS. Normalized mucosa in sinuses was postoperatively confirmed by CT and endoscopy in all patients. Patients were followed for 3 to 12 months postoperatively; the follow-up period ranged from 4 to 24 months (average, 12 months).

Statistical analysis was performed using the Mann-Whitney U test or Fisher's exact test for the duration of low-dose CAM. CT scores were compared using Cochran-Cox, Welch t, and Kruskal-Wallis H test. A P-value of $<0.05$ was considered statistically significant.

This research received no specific grant from any funding agency, commercial, or not-for-profit entity. The authors assert that all procedures contributing to this work comply with the ethical standards of the relevant national and institutional guidelines on human experimentation and with the Helsinki Declaration of 1975, as revised in 2008.

\section{Results}

The study of the patients was summarized in Table 1 . The mean age at the time of FESS was 59 years, with a range of 14 to 84 years. Most cases of bacterial culture were S. pneumonia, H. influenza, and S. aureus, including PISP (penicillin G insensitive Streptococcus pneumonia), PRSP (penicillin-resistant Streptococcus pneumonia), and BLNAR (beta-lactamase-negative ampicillin-resistant). But the results of this study were not associated with the bacterial culture. Postoperative low-dose CAM therapy was given to 29 of 41 (71\%) patients. The mean duration of low-dose CAM therapy after FESS was 36 days (range, 7 to 122 days; median, 25 days). CAM (400 mg/day) followed by low-dose CAM (200 mg/day) was used in 13 patients, and GRNX followed by lowdose CAM was used in 16 patients (Table 2, Table 3). Five patients received GRNX and no low-dose CAM (Table 2, Table 3), and 7 patients did not receive any postoperative antibiotics (control group, Table 1, Table 2). The duration of GRNX therapy varied from 2 to 7 days. There were no differences in CT scores among these groups (Cochran-Cox, Welch t, and Kruskal-Wallis H test, Table 2).

Table 1. Summary of the patients.

\begin{tabular}{ccccccc}
\hline \multicolumn{2}{c}{ Post-Ope therapy } & \multicolumn{2}{c}{ Gender } & \multicolumn{2}{c}{ Polyp } & \multicolumn{2}{c}{ Total } \\
\hline First-line & Second-line & Man & Women & With & Without & No. of case \\
\hline CAM (200 mg/day) & CAM (100 mg/day) & 8 & 5 & 3 & 10 & 13 \\
GRNX & CAM (100 mg/day) & 10 & 6 & 8 & 8 & 16 \\
GRNX & None & 4 & 1 & 4 & 1 & 5 \\
None & None & 6 & 1 & 5 & 2 & 7 \\
Total & & 28 & 13 & 20 & 21 & 41 \\
\hline
\end{tabular}

Abbreviations: CAM, clarithromycin; GRNX, garenoxacin.

Table 2. Postoperative therapy and CT score.

\begin{tabular}{|c|c|c|c|c|c|c|c|c|}
\hline \multicolumn{2}{|c|}{ Post-Ope therapy } & \multirow{2}{*}{ No. of case } & \multicolumn{6}{|c|}{ CT Score } \\
\hline First-line & Second-line & & Min & Max & Median & Mean & SD & SE \\
\hline CAM (200 mg/day) & CAM (100 mg/day) & 13 & 2 & 15 & 6 & 7 & 4.5 & 1.2 \\
\hline GRNX & CAM (100 mg/day) & 16 & 4 & 15 & 6 & 6.8 & 3.1 & 0.8 \\
\hline GRNX & None & 5 & 2 & 11 & 6 & 6.2 & 3.5 & 1.6 \\
\hline None & None & 7 & 3 & 10 & 6 & 6.1 & 2.4 & 0,9 \\
\hline Total & & 41 & 2 & 15 & 6 & 6.1 & 3.1 & 0.4 \\
\hline
\end{tabular}

P > 0.05. Abbreviations: CAM, clarithromycin; GRNX, garenoxacin; CT, computed tomography (CT scores are by the Lund-Mackay System); SD, standard deviation; SE, standard error. 
Table 3. Postoperative therapy and low-CAM period.

\begin{tabular}{|c|c|c|c|c|c|c|c|c|}
\hline \multicolumn{2}{|c|}{ Post-Ope therapy } & \multirow{2}{*}{ No. of case } & \multicolumn{6}{|c|}{ Low-CAM period (days) } \\
\hline First-line & Second-line & & Min & Max & Median & Mean & SD & SE \\
\hline CAM (200 mg/day) & CAM (100 mg/day) & 13 & 7 & 122 & 21 & 46 & 46.9 & 13 \\
\hline GRNX & CAM (100 mg/day) & 16 & 14 & 60 & 28 & 28 & 12 & 3 \\
\hline GRNX & None & 5 & 0 & 0 & 0 & 0 & 0 & 0 \\
\hline None & None & 7 & 0 & 0 & 0 & 0 & 0 & 0 \\
\hline Total & & 41 & & & & & & \\
\hline
\end{tabular}

Abbreviations: CAM, clarithromycin; GRNX, garenoxacin; SD, standard deviation; SE, standard error.

Postoperative low-dose CAM therapy was not necessary in 12 of 41 (29\%) patients, all of whom exhibited normalized sinuses mucosa within 3 months.

The second-line, low-dose CAM therapy patients $(\mathrm{n}=29)$ were divided into two groups based on the first-line antibiotic choice, a GRNX group $(\mathrm{n}=16)$ and a non-GRNX group $(\mathrm{n}=13)$.

The duration of low-dose CAM therapy ranged from 14 to 60 days (mean, 28 days) in the GRNX group and from 7 to 122 days (mean 46 days) in the non-GRNX group (Table 3 and Figure 1). The duration of low-dose CAM therapy was longer in the non-GRNX group than in the GRNX group, but the difference was not statistically significant (Mann-Whitney U test, P > 0.05, Figure 1). All cases of the postoperative CT scores were finally 0 (zero).

\section{Discussion}

It has been found that prophylactic administration of antibiotics for patients undergoing FESS can decrease postoperative morbidity and reduce the risk of infection [9].

In the early 1990s in Japan, long-term low-dose erythromycin treatment was used primarily. Since then, the anti-inflammatory effect of macrolides in vitro has been well-documented [2] [3], and macrolide therapy is now the standard treatment for CRS after FESS in Japan. Although Japanese guidelines for CRS have recommended postoperative macrolide therapy in order to obtain a favorable outcome in sinus surgery, the appropriate duration of therapy is not obvious [10]. In 1995, the recommended duration for an effective long-term low-dose erythromycin treatment after FESS, varied from 3 to 6 months [3]. More recently (2013), it has been recommended that CRS patients with rhinorrhea or postnasal drip should be treated with low-dose CAM for 6 months after FESS [4]. However, some authors have reported shorter durations, such as 2 to 3 weeks, of postoperative antibiotic treatments [11]-[13]. In accordance with these reports, and based on our own experience, there have been patients showing favorable outcomes after surgery for CRS with no postoperative antibiotic treatment. Furthermore, in terms of increasing antibiotic resistance, patients undergoing FESS for CRS have usually undergone, repeated courses of antibiotic therapy before surgery, and a previous report has shown that up to $90 \%$ of CRS patients, undergoing FESS harbor penicillin-resistant bacteria, and 65\% harbor cephalosporin-resistant species [5]. Therefore, concerns of an increasing incidence of macrolide-resistant bacterial strains should be taken seriously, and we believe that these concerns, should prompt reduced antibiotic utilization in patients with CRS after FESS.

In this retrospective study, first-line (CAM or GRNX) and second-line (low-dose CAM) postoperative antibiotic treatment regimens were established. Low-dose CAM therapy was prescribed as the second-line treatment at outpatient visits after FESS in patients for whom, the first-line may not work adequately, and it was not necessary in $29 \%$ of patients. The sinus mucosa was normalized within 3 months in all of those patients. This result indicates that patients should not routinely be given a prescription for CAM after FESS. Although previous reports have recommended a duration of 3 to 6 months for low-dose macrolide therapy [3] [4], in the present study the mean duration of low-dose macrolide therapy was about one month (36 days).

Garenoxacin (GRNX), a synthetic des-F (6)-quinolone, has been available since 2007 in Japan [14]. GRNX is reported to have anti-inflammatory activity along with strong antibacterial activity against both gram-positive and gram-negative bacteria. In one only study, the in vitro activity of GRNX against Streptococcus pneumonia was $32 \times$ that of levofloxacin and ciprofloxacin [15]. In the present study, patients who received GRNX as first- 

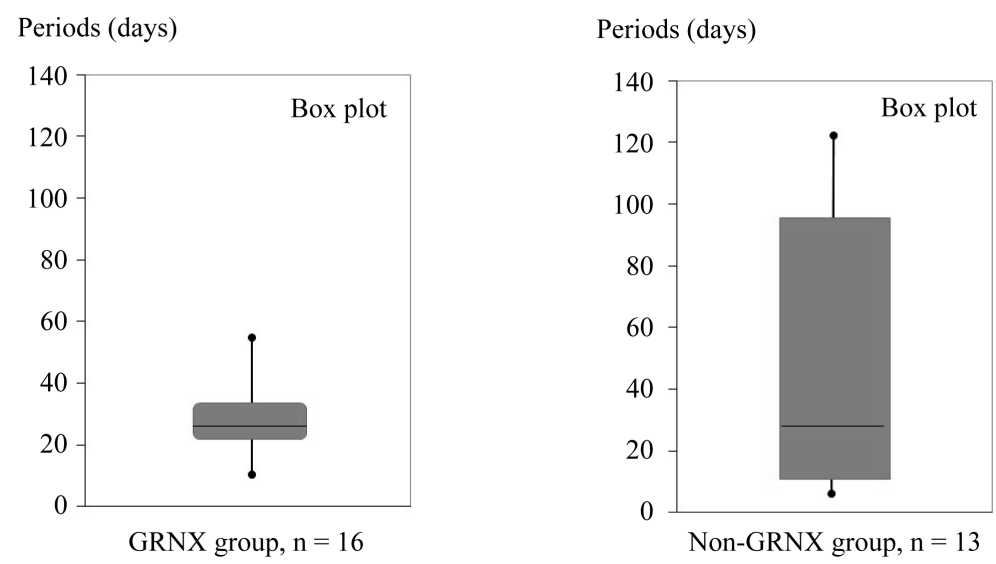

Figure 1. Duration of low-dose clarithromycin (CAM) therapy after functional endoscopic sinus surgery (FESS) in garenoxacin (GRNX) and non-GRNX groups (box-plot). The duration of therapy was longer in the non-GRNX group, but the difference was not significant $(\mathrm{P}>0.05)$.

line therapy after FESS had shorter periods of low-dose CAM therapy as outpatients, and 5 of 21 (24\%) patients who received GRNX therapy received no low-dose CAM therapy. There were no patients in the GRNX group who received low-dose CAM therapy for more than 60 days. These results suggest that GRNX could be useful for permitting shorter durations of low-dose CAM therapy postoperatively in CRS patients undergoing FESS.

\section{Conclusion}

Because of a small number of patients and the retrospective study, this report was a preliminary result, but it showed that $29 \%$ of patients did not need low-dose CAM therapy after FESS. Therefore, CAM antibiotics should not be prescribed routinely after FESS. GRNX was associated with shorter durations of low-dose CAM therapy postoperatively. The duration of CAM therapy after GRNX ranged from 14 to 60 (mean, 28) days.

\section{References}

[1] Bandhauer, F., Buhl, D. and Grossenbacher, R. (2002) Antibiotic Prophylaxis in Rhinosurgery. American Journal of Rhinology, 16, 135-139.

[2] Cervin, A. and Wallwork, B. (2007) Macrolide Therapy of Chronic Rhinosinusitis. Rhinology, 45, 259-267.

[3] Moriyama, H., Yanagi, K., Ohtori, N. and Fukami, M. (1995) Evaluation of Endoscopic Sinus Surgery for Chronic Sinusitis: Post-Operative Erythromycin Therapy. Rhinology, 33, 166-170.

[4] Nakamura, Y., Suzuki, M., Yokota, M., Ozaki, S., Ohno, N., et al. (2013) Optimal Duration of Macrolide Treatment for Chronic Sinusitis after Endoscopic Sinus Surgery. ANS, 40, 366-372.

[5] Shikani, A.H. (1996) Use of Antibiotics for Expansion of the Merocel Parking Following Endoscopic Sinus Surgery. Ear, Nose Throat Journal, 75, 524-526.

[6] Bhattacharyya, N. and Kepnes, L.J. (2008) Assessment of Trends in Antimicrobial Resistance in Chronic Rhinosinusitis. Annals of Otology, Rhinology Laryngology, 117, 448-452. http://dx.doi.org/10.1177/000348940811700608

[7] Lund, V.J. and Mackay, I.S. (1993) Staging in Rhinosinusitis. Rhinology, 107, 183-184.

[8] Kennedy, D.W., Zinreich, S.J., Shaalan, H., Kuhn, F., Naclerio, R., Loch, E.L. (1987) Endoscopic Middle Meatal Antrostomy: Theory, Technique, and Patency. Laryngoscope, 97, 1-9. http://dx.doi.org/10.1288/00005537-198708002-00001

[9] Bandhauer, F., Buhl, D. and Grossenbacher, R. (2002) Antibiotic Prophylaxis in Rhinosurgery. American Journal of Rhinology, 16, 135-139.

[10] Japan Rhinologic Society (2013) Japanese Guideline for Chronic Sinusitis. Vol. 4, Kanehara, Tokyo, 50-60.

[11] Albu, S. and Lucaciu, R. (2010) Prophylactic Antibiotics in Endoscopic Sinus Surgery: A Short Follow-Up Study. American Journal of Rhinology Allergy, 24, 306-309. http://dx.doi.org/10.2500/ajra.2010.24.3475

[12] Jiang, R.S., Liang, K.L., Yang, K.Y., Shiao, J.Y., Su, M.C., et al. (2008) Postoperative Antibiotic Care after Functional 
Endoscopic Sinus Surgery. American Journal of Rhinology, 22, 608-612. http://dx.doi.org/10.2500/ajr.2008.22.3241

[13] Bhandarkar, N.D., Mace, J.C. and Smith, T.L. (2011) Endoscopic Sinus Surgery Reduces Antibiotic Utilization in Rhinosinusitis. International Forum of Allergy Rhinology, 1, 18-22.

[14] Takahata, M., Shimakura, M., Hori, R., Kizawa, K., Todo, Y., et al. (2001) In Vitro and in Vivo Antimicrobial Activities of T-3811ME (BMS-284756) against Mycoplasma Pneumonia. Antimicrobial Agents and Chemotherapy, 45, 312315. http://dx.doi.org/10.1128/AAC.45.1.312-315.2001

[15] Ito, M., Maruyama, Y., Murono, S., Wakisaka, N., Kondo, S., et al. (2012) Efficacy and Safety of Garenoxacin in the Treatment of Upper Respiratory Tract Infections. ANL, 39, 512-518. 
Scientific Research Publishing (SCIRP) is one of the largest Open Access journal publishers. It is currently publishing more than 200 open access, online, peer-reviewed journals covering a wide range of academic disciplines. SCIRP serves the worldwide academic communities and contributes to the progress and application of science with its publication.

Other selected journals from SCIRP are listed as below. Submit your manuscript to us via either submit@scirp.org or Online Submission Portal.
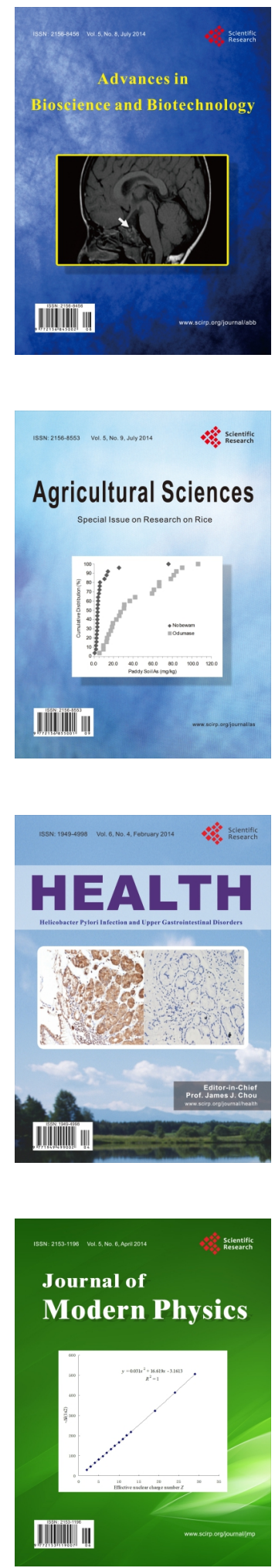
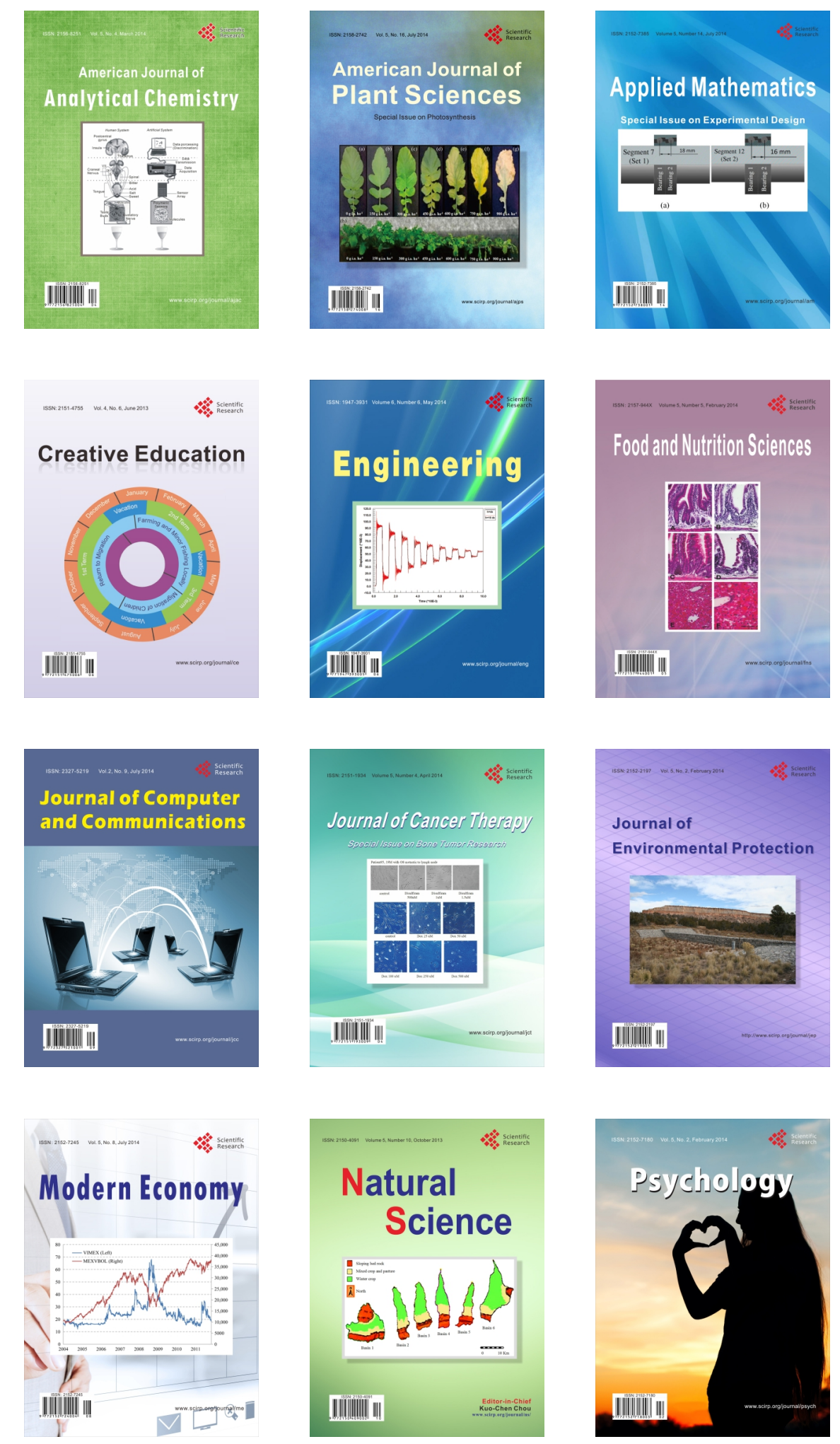